\title{
Evolution in Diagnosis and Classification of Diabetes
}

\author{
L. Sreenivasamurthy \\ Department of Medicine, Dr B.R. Ambedkar Medical College, Bangalore, India \\ Email: drsreenivasamurthy@gmail.com
}

How to cite this paper: Sreenivasamurthy, L. (2021) Evolution in Diagnosis and Classification of Diabetes. Journal of Diabetes Mellitus, 11, 200-207.

https://doi.org/10.4236/jdm.2021.115017

Received: October 22, 2021

Accepted: November 13, 2021

Published: November 16, 2021

Copyright () 2021 by author(s) and Scientific Research Publishing Inc. This work is licensed under the Creative Commons Attribution International License (CC BY 4.0).

http://creativecommons.org/licenses/by/4.0/ (c) (i) Open Access

\begin{abstract}
Diabetes, has been known to mankind for centuries, often described by its unique nature of sweet tasting urine or by the symptoms of excessive urination and thirst. Several renowned scientists have attempted to describe the symptoms, pathophysiology, diagnosis and various experimental treatment options with varying degrees of success. The current widely accepted classification focuses on etiology and pathogenesis to guide treatment and is very successful and adapted across the world. Diagnostic criteria underwent multiple changes to define glycemic targets which were more and more guided by better understanding of the disease pathogenesis. Recent research focuses on a more individualized approach to managing diabetes, better understanding and identifying individuals who are at an increased risk of complications at diagnosis itself to offer more personalized management to prevent long term complications. Early differentiation of individual based on multiple factors rather than a single parameter is the path being paved currently. BMI, Waist circumference, age at diagnosis, $\mathrm{HbAlc}$, triglycerides, HDL cholesterol, GAD Antibodies, Homeostasis model assessment of Insulin Resistance (HOMA-2 IR), beta-cell dysfunction (HOMA2-Beta), and fasting and stimulated C-peptide, are core parameters in the current rationalized approach towards better long term outcomes for people with diabetes. Cluster based identification and focused treatment with individualistic targets is the way forward in Diabetes management.
\end{abstract}

\section{Keywords}

Diabetes HbA1c Clusters GAD Antibodies C Peptide

\section{Introduction}

It was in the early $5^{\text {th }}$ and $6^{\text {th }}$ century that diabetes was first noted by Ayurvedic scientists Sushruta and Charaka, they defined this illness by the characteristic of sweet tasting urine, described as "Madhumeha" or "honey urine". Several dis- 
eases had polyuria as a clinical symptom and were collectively called Prameha ("to flow"). It was these Indian scientists who also first described the distinct possibility of two types of diabetes-Type 1 and Type 2 [1] [2] [3]. In the 1600s Paracelsus also described diabetes as a constitutional disease that affected the kidneys and provoked excessive urination. By 1700s several European researchers like Thomas Willis and William Cullen had attempted to describe diabetes through its classical clinical symptoms of sweet tasting urine and a disorder of the kidney. Thomas Cawley in 1788, conducted an autopsy on a diabetic patient and suggested a link between the pancreas and diabetes after observing stones and signs of tissue damage in the pancreas [4].

Research by Joseph von Mering and Oskar Minkowski on dogs led to the observation that when the pancreas of a dog is excised, it developed symptoms of diabetes. And when the urine was tested for sugar, they were able to confirm the diagnosis of diabetes. This discovery heralded the role of pancreas in diabetes [5].

Further research to understand and classify diabetes happened in the 1800s, when researchers Apollinaire Bouchardat and E. Lancereux were able to distinguish between different patient types, notable patients who had more severe symptoms, were lean and expected to have poorer outcomes along with pancreatic lesions on autopsy as against another type of patient group who had a milder form of disease, were overweight and presented at a later stage in life and expected to have a much better prognosis [6]. These descriptions are comparable to the two types identified by Ayurvedic physicians Sushruta and Charka (400-500 AD), with one type being associated with youth and the other with being overweight. Harold Percival Himsworth in 1936 brought forth a clearer distinction, differentiating two types of diabetes based on sensitivity to insulin (both injected and pancreatic) [7]. In 1950, R. D. Lawrence observed that some diabetics were deficient in insulin and others were not [8]. Philip Hugh-Jones, while working in Jamaica in 1955, clarified Lawrence's classification and coined the terms "type 1" and "type 2" diabetes [9].

\section{Transformations in Classification of Diabetes}

Understanding diabetes has underdone massive transformations over the past century. Until the 1960s, no systematic classification of diabetes existed. It was in 1965, that an Expert Committee on Diabetes Mellitus published the first World Health Organization (WHO) report on diabetes classification [10]. This report was one of the first attempts at an International consensus on classification of diabetes. The expert committee classified diabetes based on age of recognized onset and need for insulin for survival, which seemed to be the only reliable means of classification for universal use.

The report also recognized certain specific types of diabetes including brittle, insulin-resistant, gestational, pancreatic, endocrine, and iatrogenic diabetes [9]. Since then, several pathogenic mechanisms have been described and long-term studies have shown different courses and outcomes of different types of diabetes.

The National Diabetes Data Group (NDDG) formulated a revised classifica- 
tion of glucose intolerance that was later amended and adopted in the second report by WHO Expert Committee in 1980 [11] [12]. The 1980 Expert Committee proposed two major classes of diabetes mellitus and named them insulin-dependent diabetes mellitus (IDDM) or type 1, and non-insulin-dependent diabetes mellitus (NIDDM) or type 2 [12]. In the 1985 Study Group Report, the terms type 1 and type 2 were omitted, but the classes IDDM and NIDDM were retained, and a new class of malnutrition-related diabetes mellitus (MRDM) was introduced [13]. It was the 1985 WHO classification that was essentially based on clinical descriptions, with specific focus on the pharmacologic management of patients (i.e., insulin-dependent, non-insulin-dependent, gestational). Both the reports from WHO included other types of diabetes and impaired glucose tolerance (IGT) as well as gestational diabetes mellitus (GDM). The 1985 classification was widely accepted and used internationally and represented a compromise between clinical and etiological classifications. This classification essentially helped clinicians to manage diabetes irrespective of the etiological origin.

In 1999, the WHO incorporated an approach developed by Kuzuya and Matsuda [14], which clearly separated the criteria based on etiology from those related to the degree of deficiency of insulin or insulin action and defined each patient on the basis of these two sets of criteria. It is now well established that diabetes may progress through several clinical stages during its natural history, quite independent of its etiology. Individuals may move from one stage to another stage in both directions. Even if there is no information concerning the underlying etiology, persons who are developing the disease can be categorized by stage according to clinical characteristics. This type of classification allows for various degrees of hyperglycemia in individuals irrespective of the disease process. The glycemic stages range from normoglycemia (normal glucose tolerance) to hyperglycemia where insulin is required for survival [15]. The stage of glycemia may change over time depending on the extent of the underlying disease processes.

Over the years, the terms "insulin-dependent diabetes mellitus (IDDM)," and "noninsulin-dependent diabetes mellitus (NIDDM)" have been removed from classifications as they were more confusing for clinicians, as these were based on the treatment given and age rather than the pathogenesis. In the current widely used classification, the terms "Type 1" and "Type 2" are retained. As per the latest ADA 2021 classification, Diabetes can be classified into the following general categories based on etiology [16]: Type 1 diabetes (due to autoimmune b-cell destruction, usually leading to absolute insulin deficiency, including latent autoimmune diabetes of adulthood), Type 2 diabetes (due to a progressive loss of adequate b-cell insulin secretion frequently on the background of insulin resistance), specific types of diabetes due to other causes, e.g., monogenic diabetes syndromes (such as neonatal diabetes and maturity-onset diabetes of the young), diseases of the exocrine pancreas (such as cystic fibrosis and pancreatitis), and drug- or chemical-induced diabetes (such as with glucocorticoid use, in the treatment of HIV/AIDS, or after organ transplantation), and Gestational diabetes mellitus 
(diabetes diagnosed in the second or third trimester of pregnancy that was not clearly overt diabetes prior to gestation) [16].

\section{Evolution in Diagnostic Criteria over the Decades}

The guidance towards diagnostic criteria for diabetes has also undergone changes over the past few decades, in 1979 the US Diabetes Data Group (also known as the NDDG) upon reviewing epidemiological studies observed a correlation between diabetes related complications like nephropathy and retinopathy and blood glucose values, which then determined their diagnostic blood glucose targets [11]. Diagnostic blood glucose level was fixed at $140 \mathrm{mg} / \mathrm{dl}(7.78 \mathrm{mmol} / \mathrm{l})$, if glycaemia was $<140 \mathrm{mg} / \mathrm{dl}$ but $>110 \mathrm{mg} / \mathrm{dl}$, an OGTT was required (diagnostic if glycaemia $>200 \mathrm{mg} / \mathrm{dl}$ two hours post-OGTT) [17]. The WHO also adopted this in 1980 with a few changes [17]. Towards 1997, the expert committee from ADA revised the criteria for diagnosis: the cut-off of fasting plasma glucose (FPG) was lowered from $140 \mathrm{mg} / \mathrm{dl}(7.78 \mathrm{mmol} / \mathrm{l})$ to $\geq 126 \mathrm{mg} / \mathrm{dl}(\geq 7.0 \mathrm{mmol} / \mathrm{l})$ based on population studies showing that the incidence of retinopathy was higher even with blood glucose values $>120 \mathrm{mg} / \mathrm{dl}$ [18]. Moreover, a cut off value of 126 $\mathrm{mg} / \mathrm{dl}$ for FPG reduces the discrepancy between FPG and 2 hours OGTT blood glucose values, promoting its use in place of OGTT, which is associated with technical challenges, lower reproducibility and greater costs [18]. The European DECODE study (Diabetes Epidemiology: Collaborative Analysis of Diagnostic Criteria in Europe) did show that two hours post-OGTT blood glucose is more predictive than FPG in relation to cardiovascular disease [19]. There is no clear guidance as to which is the best diagnostic test for diabetes, but the general consensus is that FPG remains an ideal diagnostic test in clinical practice and OGTT would be useful in research studies or in clinical situations that require a clear distinction between diabetes and other aberrations of glucose metabolism. The ADA defined new diagnostic criteria within diabetes referring to IFG (Impaired Fasting Glucose) for fasting blood glucose between 110 and $125 \mathrm{mg} / \mathrm{dl}$ and IGT (Impaired Glucose Tolerance) defined as blood sugar between 140 and $199 \mathrm{mg} / \mathrm{dl}$ at two hours post OGTT [20]. Such evaluations of two blood glucose abnormalities do not indicate two defined clinical conditions, but more of a risk towards possible evolution towards diabetes itself and, apart from diabetes, a risk of cardiovascular complications. HbAlc of $>6.5 \%$ is part of the diagnostic criteria for diabetes, however it is posed with many challenges because of its susceptibility to vary based on different clinical conditions such as hemodialysis, pregnancy, HIV treatment, age, race/ethnicity, pregnancy status, genetic background, and anemia/hemoglobinopathies. There are several other diagnostic markers (i.e., antibodies, C-Peptide levels, serum or plasma insulin, and genetic testing for MODY etc.) which are very specific towards identifying the variants in diabetes, however such parameters are limited in its applicability in resource constrained settings and countries especially due to their limited availability and affordability.

The current ADA diagnostic Criteria for diabetes and prediabetes and gestational diabetes are mentioned in Tables 1-3 respectively [16]: 
Table 1. Criteria for the diagnosis of diabetes.

FPG $\geq 126 \mathrm{mg} / \mathrm{dL}(7.0 \mathrm{mmol} / \mathrm{L})$ after an overnight fast. Fasting is defined as no caloric intake for at least $8 \mathrm{~h} .^{*}$

OR

2-h PG $\geq 200 \mathrm{mg} / \mathrm{dL}$ (11.1 mmol/L) during OGTT. The test should be performed as described by WHO, using a glucose load containing the equivalent of $75 \mathrm{~g}$ anhydrous glucose dissolved in water. ${ }^{*}$

OR

A1C $\geq 6.5 \%$ ( $48 \mathrm{mmol} / \mathrm{mol})$. The test should be performed in a laboratory using a method that is NGSP certified and standardized to the DCCT assay.*

OR

In a patient with classic symptoms of hyperglycemia or hyperglycaemic crisis, a random plasma glucose $\geq 200 \mathrm{mg} / \mathrm{dL}$ ( $11.1 \mathrm{mmol} / \mathrm{L})$.

DCCT, Diabetes Control and Complications Trial; FPG, fasting plasma glucose; OGTT, oral glucose tolerance test; WHO, World Health Organization; 2-h PG, 2-h plasma glucose. ${ }^{\star}$ In the absence of unequivocal hyperglycemia, diagnosis requires two abnormal test results from the same sample or in two separate test samples.

Table 2. Criteria defining prediabetes*.

FPG $100 \mathrm{mg} / \mathrm{dl}(5.6 \mathrm{mmol} / \mathrm{L})$ to $125 \mathrm{mg} / \mathrm{dl}(6.9 \mathrm{mmol} / \mathrm{L})(\mathrm{IFG})$

OR

2-h PG during 75-g OGTT $140 \mathrm{mg} / \mathrm{dl}(7.8 \mathrm{mmol} / \mathrm{L})$ to $199 \mathrm{mg} / \mathrm{dl}(11.0 \mathrm{mmol} / \mathrm{L})(\mathrm{IGT})$

OR

A1C $5.7 \%-6.4 \%(39-47 \mathrm{mmol} / \mathrm{mol})$

FPG, fasting plasma glucose; IFG, impaired fasting glucose; IGT, impaired glucose tolerance; OGTT, oral glucose tolerance testing; 2-h PG, 2-h plasma glucose. ${ }^{\star}$ For all three tests, risk is continuous, extending below the lower limit of the range and becoming disproportionately greater at the higher end of the range.

Table 3. Screening for and diagnosis of GDM.

One-step strategy:

Perform a 75-g OGTT, with plasma glucose measurement when patient is fasting and at 1 and $2 \mathrm{~h}$, at 24 - 28 weeks gestation in women not previously diagnosed with diabetes.

The OGTT should be performed in the morning after an overnight fast of at least $8 \mathrm{~h}$. The diagnosis of GDM is made when any of the following plasma glucose values are met or exceeded:

- Fasting: $92 \mathrm{mg} / \mathrm{dl}(5.1 \mathrm{mmol} / \mathrm{L})$

- $1 \mathrm{~h}: 180 \mathrm{mg} / \mathrm{dl}(10.0 \mathrm{mmol} / \mathrm{L})$

- 2 h: $153 \mathrm{mg} / \mathrm{dl}(8.5 \mathrm{mmol} / \mathrm{L})$

Two-step strategy:

Step 1: Perform a 50-g GLT (nonfasting), with plasma glucose measurement at $1 \mathrm{~h}$, at 24 - 28 weeks of gestation in women not previously diagnosed with diabetes.

If the plasma glucose level measured $1 \mathrm{~h}$ after the load is $\geq 130,135$, or $140 \mathrm{mg} / \mathrm{dl}(7.2,7.5$, or $7.8 \mathrm{mmol} / \mathrm{L}$, respectively), proceed to 100-g OGTT.

Step 2: The 100-g OGTT should be performed when the patient is fasting.

The diagnosis of GDM is made when at least two ${ }^{*}$ of the following four plasma glucose levels (measured fasting and at 1,2 , and $3 \mathrm{~h}$ during OGTT) are met or exceeded (Carpenter-Coustan criteria [193]):

- Fasting: $95 \mathrm{mg} / \mathrm{dl}(5.3 \mathrm{mmol} / \mathrm{L})$

- $1 \mathrm{~h}: 180 \mathrm{mg} / \mathrm{dl}(10.0 \mathrm{mmol} / \mathrm{L})$

- $2 \mathrm{~h}: 155 \mathrm{mg} / \mathrm{dl}(8.6 \mathrm{mmol} / \mathrm{L})$

- $3 \mathrm{~h}: 140 \mathrm{mg} / \mathrm{dl}(7.8 \mathrm{mmol} / \mathrm{L})$

GDM, gestational diabetes mellitus; GLT, glucose load test; OGTT, oral glucose tolerace test. *American College of Obstetricians and Gynecologists notes that one elevated value can be used for diagnosis. 


\section{Evolution in Diagnosis and Understanding Diabetes}

With ongoing research into the heterogeneous nature of type 2 diabetes etiology and progression, there is an increasing need for a more individualistic approach to managing type 2 diabetes, redefining classification to identify individuals who are at an increased risk of complications at diagnosis itself so as to offer more personalized management to prevent long term complications. Research by Emma Ahlquist and colleagues' remark that the current treatment guidelines focused on measurement of a single parameter of glucose alone has major limitations in the fact that they respond to poor metabolic control as it develops [21]. Moreover, they contend that there is no means to predict which patients need more intensive treatment. This is a crucial factor for prevention of long term complications of diabetes as the target tissues have metabolic memory that will remember poor metabolic control for prolonged periods of time [20]. Cluster based classification proposed by her team in the Scandinavian population advocate identifying clusters with specific characteristics based on 6 clinical variables that are measured at the time of diagnosis, namely GAD Antibodies, age at diagnosis of diabetes, Body Mass Index (BMI), Glycated Hemoglobin (HbAlc), Homeostasis model assessment of Insulin Resistance (HOMA-2 IR) and beta-cell dysfunction (HOMA2-Beta). The Custer 1 is characterized by early-onset disease, relatively low BMI, poor metabolic control, insulin deficiency, and presence of GADA labelled as severe autoimmune diabetes (SAID). Cluster 2, labelled as severe insulin-deficient diabetes (SIDD), is GADA negative but similar to cluster 1, with lower age at onset, relatively low BMI, low insulin secretion (low HOMA2-B index), and poor metabolic control. Cluster 3 labelled as severe insulin resistant diabetes (SIRD) is characterized by insulin resistance (high HOMA2-IR index) and high BMI. Cluster 4, is characterized by obesity but not by insulin resistance, and was labelled as mild obesity-related diabetes (MOD). Cluster 5 (labelled as mild age-related diabetes [MARD]) refers to patients who were older than patients in other clusters but showed only modest metabolic derangements. This kind of clinically useful stratification of diabetes may pave the way towards precision and more personalized medicine in diabetes management [21].

New and unique cluster-based classification system has also been studied in the south Asian population from India, by RM Anjana and colleagues [22]. The Asian phenotype is classically different with unique characteristics like younger age of onset, thin-fat Indian phenotype, low muscle mass, increased insulin resistance, and less obesity. The 8 variables included defining the clusters were age at diagnosis, BMI, waist circumference, $\mathrm{HbAlc}$, triglycerides, $\mathrm{HDL}$ cholesterol as well as fasting and stimulated C-peptide. Two clusters identified were similar to those in the Scandinavian population: SIDD and MARD and two new groups IROD (Insulin Resistant Obese Diabetes) and CIRDD (Combined Insulin Resistant and Deficient Diabetes) were identified [22].

These advances in the identification, diagnosis and classification of diabetes 
have major implications in revolutionizing management of diabetes. Individuals falling in the milder clusters would not require aggressive management whereas individuals with severe insulin resistance and insulin deficiency would benefit immensely from early aggressive management with pharmacological agents as well as more frequent screening for development of complications of diabetes. Such cluster-based management also aids in appropriate selection of drugs based on the individual specific requirements.

In essence, research from across the world has guided us to more in-depth understanding of this complex and chronic disease. Moreover, the research has led to adaptable and adoptable advanced treatment guidelines and targets aimed towards a more precise and personalized management than presently recommended, "an ideal path forward".

Nevertheless, the challenge is overwhelming in resource-constrained and limited health expenditure settings such as the developing countries like India and China, where the prevalence of Diabetes is exponentially rising. Therefore, the adoption of more complex and multiple pathways for diagnosis and management may not be affordable and hence practical and feasible. Hence, simple diagnosis with parameters like HbA1c, FPG and OGTT might still be the most suitable option for the large patient populations in these countries.

\section{Conflicts of Interest}

The author declares no conflicts of interest regarding the publication of this paper.

\section{References}

[1] MacFarlane, I.A. (1990) Mathew Dobson of Liverpool (1735-1784) and the History of Diabetes. Practical Diabetes International, 7, 246-248. https://doi.org/10.1002/pdi.1960070603

[2] Frank, L.L. (1957) Diabetes Mellitus in the Texts of Old Hindu Medicine (Charaka, Susruta, Vagbhata). The American Journal of Gastroenterology, 27, 76-95.

[3] Roy, C.K., Ojha, J.K. and Bajpai, H.S. (1993) A Review of the History of Prameha and Diabetes Mellitus. Ancient Science of Life, 12, 394-398.

[4] Stylianou, C. and Kelnar, C. (2009) The Introduction of Successful Treatment of Diabetes Mellitus with Insulin. Journal of the Royal Society of Medicine, 102, 298-303. https://doi.org/10.1258/jrsm.2009.09k035

[5] Busnardo, A.C., DiDio, L.J.A., Tidrick, R.T. and Thomford, N.R. (1983) History of the Pancreas. The American Journal of Surgery, 146, 539-550. https://doi.org/10.1016/0002-9610(83)90286-6

[6] Rachmiel, R. (1979) The Endocrine Pancreas, Past and Present. In: Klachko, D.M., Anderson, R.R., Burns, T.W. and Werner, H.V., Eds., The Endocrine Pancreas and Juvenile Diabetes, Springer Science \& Business Media, Berlin, 2-3.

[7] Himsworth (1936) Diabetes Mellitus: Its Differentiation into Insulin-Sensitive and Insulin-Insensitive Types. The Lancet, 227, 127-130. https://doi.org/10.1016/S0140-6736(01)36134-2

[8] Oakley, W.G. (1969) R. D. Lawrence, M.D., F.R.C.P., 1892-1968. Diabetes, 18, 54-55. 
https://doi.org/10.2337/diab.18.1.54

[9] Hugh-Jones, P. (1955) Diabetes in Jamaica. The Lancet, 266, 891-897. https://doi.org/10.1016/S0140-6736(55)92530-7

[10] World Health Organization WHO (1965) Diabetes Mellitus. Report of a WHO Expert Committee. Technical Report Series 310. WHO, Geneva.

[11] National Diabetes Data Group (1979) Classification and Diagnosis of Diabetes Mellitus and Other Categories of Glucose Intolerance. Diabetes, 28, 1039-1057. https://doi.org/10.2337/diab.28.12.1039

[12] World Health Organization (1980) WHO Expert Committee on Diabetes Mellitus. Second Report. Technical Report Series 646. WHO, Geneva.

[13] World Health Organization (1985) Diabetes Mellitus. Report of a WHO Study Group. Technical Report Series 727. WHO, Geneva, 727.

[14] Kuzuya, T. and Matsuda, A. (1997) Classification of Diabetes on the Basis of Etiologies versus Degree of Insulin Deficiency. Diabetes Care, 20, 219-220. https://doi.org/10.2337/diacare.20.2.219

[15] World Health Organization (1999) Definition, Diagnosis and Classification of Diabetes Mellitus and Its Complications. Part 1: Diagnosis and Classification of Diabetes Mellitus. WHO, Geneva, Report No. WHO/NCD/NCS/99.2.

[16] American Diabetes Association (2021) Classification and Diagnosis of Diabetes: Standards of Medical Care in Diabetes-2021. Diabetes Care, 44, S15-S33. https://doi.org/10.2337/dc21-S002

[17] Expert Committee on Diabetes Mellitus (1980) Second Report. Technical Report Series 646. World Health Organization, Geneva.

[18] The Expert Committee on the Diagnosis and Classification of Diabetes Mellitus (1997) Report of the Expert Committee on the Diagnosis and Classification of Diabetes. Diabetes Care, 20, 1183-1197. https://doi.org/10.2337/diacare.20.7.1183

[19] DECODE Study Group on Behalf of the European Diabetes Epidemiology Group (1999) Glucose Tolerance and Mortality: Comparison of WHO and American Diabetes Association Diagnostic Criteria. The Lancet, 354, 617. https://doi.org/10.1016/S0140-6736(98)12131-1

[20] Rivellese, A.A., Bozzetto, L. and Massaro, P. (2007) Advances in Diabetes Diagnostics. European Endocrine Disease, No. 1, 20-22. https://doi.org/10.17925/EE.2007.00.01.20

[21] Ahlqvist, E., Storm, P., Käräjämäki, A., Martinell, M., Dorkhan, M., Carlsson, A., Vikman, P., Prasad, R.B., Aly, D.M., Almgren, P., Wessman, Y., Shaat, N., Spégel, P., Mulder, H., Lindholm, E., Melander, O., Hansson, O., Malmqvist, U., Lernmark, Å., Lahti, K., Forsén, T., Tuomi, T., Rosengren, A.H. and Groop, L. (2018) Novel Subgroups of Adult-Onset Diabetes and Their Association with Outcomes: A Data-Driven Cluster Analysis of Six Variables. The Lancet Diabetes \& Endocrinology, 6, 361-369. https://doi.org/10.1016/S2213-8587(18)30051-2

[22] Anjana, R.M., Pradeepa, R., Unnikrishnan, R., Tiwaskar, M., Aravind, S.R., Saboo, B., Joshi, S.R. and Mohan, V. (2021) New and Unique Clusters of Type 2 Diabetes Identified in Indians. Journal of the Association of Physicians of India, 69, 58-61. 